\title{
A SIGNAL ADAPTIVE ARRAY INTERPOLATION APPROACH WITH REDUCED TRANSFORMATION BIAS FOR DOA ESTIMATION OF HIGHLY CORRELATED SIGNALS
}

\author{
Marco A. M. Marinho ${ }^{\star \dagger} \quad$ Felix Antreich ${ }^{\star} \quad$ João Paulo. C. L. da Costa ${ }^{\dagger} \quad$ Josef A. Nossek ${ }^{\ddagger}$ \\ * German Aerospace Center (DLR), Inst. for Communications and Navigation, 82234 Wessling, Germany \\ ${ }^{\dagger}$ Dept. of Electrical Engineering, Universidade de Brasília (UnB), Brasília, Brazil \\ ${ }^{\ddagger}$ Inst. for Circuit Theory \& Signal Processing, Munich University of Technology (TUM), Germany \\ email: marco.marinho@ieee.org ${ }^{\star \dagger}$, felix.antreich@dlr.de ${ }^{\star}$, joaopaulo.dacosta@ene.unb.br ${ }^{\dagger}$, nossek@nws.ei.tum.de ${ }^{\ddagger}$
}

\begin{abstract}
Sensor arrays with Vandermonde or centro-hermitian responses cannot always be constructed. However, such array response structure can be achieved by means of a mapping which transforms the real array response to an array response with the desired properties by applying array interpolation algorithms.

In this work a low-complexity, multi-sector, signal adaptive array interpolation approach that achieves low transformation bias in the presence of highly correlated signals is presented. Estimation of Signal Parameters via Rotational Invariance (ESPRIT) algorithm with Forward Backward Average (FBA) and Spatial Smoothing (SPS) as well as model order estimation is applied after array interpolation in conjunction with the Vandermonde Invariance Transformation (VIT) to obtain precise high resolution estimates in closed form. A set of numerical simulations show that the proposed approach provides precise estimates for arbitrary array responses in highly correlated signal signal environments.
\end{abstract}

Index Terms - Array Interpolation, Array Mapping, Antenna Arrays, Vandermonde Invariance Transformation

\section{INTRODUCTION}

Vandermonde or centro-hermitian array structures are of special interest in DOA estimation since they allow for reduced computational complexity with fast converging methods or even closed-form solutions. Popular methods such as Iterative Quadratic Maximum Likelihood (IQML) [1], Root-WSF [2] and Root-MUSIC [3] all rely on a Vandermonde or centro-hermitian array response. Another important property a centro-hermitian array response is allowing the application Spatial Smoothing (SPS) [4] and Forward Backward Averaging (FBA) [5]. These techniques enable the application of subspace based DOA estimation methods and precise model order estimation in the presence of highly correlated or even coherent signals. Dealing with highly correlated signals is of great importance when facing strong multipath scenarios or in case of safety-critical applications when specific jamming, meaconing, or spoofing is received.

To obtain an array response that is Vandermonde or centrohermitian is very hard in reality due to effects such as mutual coupling of the antennas, changes in antenna location, material tolerances, hardware biases, and the surrounding environment of the array. Even when the construction is possible there is no guarantee that the response of such an array will be kept invariant over time, e.g. due to wear and temperature stability. A solution to these limitations array interpolation (mapping) was proposed [6] where an arbitrary array response is mapped onto the desired Vandermonde or centro-hermitian response. Most array interpolation schemes divide the complete angular region into limited angular sectors. For each sector a mapping/transformation matrix is defined using knowledge of the empirical measured array response. Then after transformation to a desired virtual array, FBA or SPS [7] and DOA estimation algorithms such as Root-MUSIC [8] can be applied. However, when performing array interpolation with a sector-by-sector processing the mapping matrices have to be carefully derived in order to minimize the transformation bias within each sector and on the other hand to control its out-of-sector response. The out-of-sector response was neglected in earlier works [7], [8], [9], [10]. Addressing the out-of-sector response by a signal adaptive weighting and a sectorby-sector estimation of highly correlated and closely spaced signal environments is proposed in [11] and [12]. Furthermore, although before the array interpolation the noise is white, after the array interpolation the noise becomes colored. Therefore, a prewhitening step is necessary for MUSIC [13] and Root-MUSIC algorithms [3],[8]. Such prewhitening would destroy the shift invariance properties necessary for the standard ESPRIT algorithm [14]. Array interpolation techniques that allow the application of a modified ESPRIT algorithm have been proposed in [15] and [16]. These techniques do not require the prewhitening step, thus allowing the direct application of the ESPRIT algorithm. However, they ignore the out-of-sector response and they do not consider the application of FBA or/and SPS and thus cannot be applied with highly correlated signals.

Another application of the array interpolation technique can be seen in [17] where the Vandermonde Invariance Transformation (VIT) was developed. The VIT does not try to address the physical imperfections of the array response but instead transforms the response of an array with a uniform Vandermonde response into one with a non uniform phase response. The VIT provides a noise shaping effect by lowering the noise power over a desired angular region and allowing a more precise DOA estimation at the cost of increased computational load.

In this work we do not apply the classical sector-by-sector mapping processing. Instead we propose a signal adaptive multisector array interpolation method that minimizes the transformation bias, allows dealing with highly correlated signals by applying FBA and/or SPS, and enables closed-form DOA estimation by ESPRIT with a generalized eigenvalue decomposition (GEVD). We derive a single mapping matrix that considers several angular sectors which include all the impinging signals of interest. These sectors are estimated and combined by applying a power scanning method of low complexity. The degrees of freedom of the mapping are distributed 
among the parts of the resulting combined sector following a simple adaptive weighting method, minimizing the transformation bias. The proposed approach avoids the out-of-sector response problems [11], [12] and allows to jointly estimate the DOAs of all impinging signals, while for the sector-by-sector processing for each sector a DOA estimation has to be performed, cf. [8], [9], [10] ,[11], [12], [15], [16]. Afterwards the VIT can be applied and followed by a second application of ESPRIT an additional gain of approx. $2 \mathrm{~dB}$ in DOA estimation performance can be achieved.

The proposed method can be applied to the vast majority of systems that rely on sensor arrays, e.g, radar systems, channel sounding and sonars. For this work we consider Global Navigation Satellite Systems (GNSS). In these systems the signal is constituted of a line of sight component and highly correlated or even coherent multipath components as well as spoofing.

\section{DATA MODEL AND ARRAY INTERPOLATION}

We consider a set of $d$ wavefronts impinging onto an antenna array composed of $M$ antenna elements displaced on a line. The received baseband signal can be expressed in matrix form as

$$
\mathbf{X}=\mathbf{A} \mathbf{S}+\mathbf{N} \in \mathbb{C}^{M \times N},
$$

where $\mathbf{S} \in \mathbb{C}^{d \times N}$ is the matrix containing the $N$ symbols transmitted by each of the $d$ sources, $\mathbf{N} \in \mathbb{C}^{M \times N}$ is the noise matrix with its entries drawn from $\mathcal{C N}\left(0, \sigma_{n}^{2}\right)$, and

$$
\mathbf{A}=\left[\mathbf{a}\left(\theta_{1}\right), \mathbf{a}\left(\theta_{2}\right), \ldots, \mathbf{a}\left(\theta_{d}\right)\right] \in \mathbb{C}^{M \times d},
$$

where $\theta_{i}$ is the azimuth angle of the $i-$ th signal and $\mathbf{a}\left(\theta_{i}\right) \in \mathbb{C}^{M \times 1}$ is the array response (empirical measurement). by

The received signal covariance matrix $\mathbf{R}_{\mathbf{X X}} \in \mathbb{C}^{M \times M}$ is given

$$
\mathbf{R}_{\mathbf{X X}}=\mathrm{E}\left\{\mathbf{X X}^{\mathrm{H}}\right\}=\mathbf{A R}_{\mathbf{S S}} \mathbf{A}^{\mathrm{H}}+\mathbf{R}_{\mathbf{N N}},
$$

where $(.)^{\mathrm{H}}$ stands for the conjugate transposition, and

$$
\mathbf{R}_{\mathbf{S S}}=\left[\begin{array}{cccc}
\sigma_{1}^{2} & \gamma_{1,2} \sigma_{1} \sigma_{2} & \cdots & \gamma_{1, d} \sigma_{1} \sigma_{d} \\
\gamma_{1,2}^{*} \sigma_{1} \sigma_{2} & \sigma_{2}^{2} & & \vdots \\
\vdots & & \ddots & \\
\gamma_{1, d}^{*} \sigma_{1} \sigma_{d} & \gamma_{2, d}^{*} \sigma_{2} \sigma_{d} & \cdots & \sigma_{d}^{2}
\end{array}\right],
$$

where $\sigma_{i}^{2}$ is the power of the $i-$ th signal and $\gamma_{a, b} \in \mathbb{C},\left|\gamma_{a, b}\right| \leq 1$ is the cross correlation coefficient between signals $a$ and $b$. $\mathbf{R}_{\mathrm{NN}} \in$ $\mathbb{C}^{M \times M}$ is a matrix with $\sigma_{n}^{2}$ over its diagonal and zeros elsewhere.

The array interpolation technique consists of finding a transformation matrix $\mathbf{B}$ that transforms the real array response $\mathbf{A}_{\mathcal{S}}$ for a given countable and discrete set of angles $\mathcal{S}$, called a sector, into the desired array response $\overline{\mathbf{A}}_{\mathcal{S}}$. Thus, the matrix $\mathbf{B}$ can be seen as the matrix that achieves the best transform between a set of vectors $\mathbf{A}_{\mathcal{S}}$ and $\overline{\mathbf{A}}_{\mathcal{S}}$. The simplest solution for obtaining $\mathbf{B}$ is a least squares fit via

$$
\mathbf{B}=\overline{\mathbf{A}}_{\mathcal{S}} \mathbf{A}_{\mathcal{S}}^{\dagger} \in \mathbb{C}^{M \times M},
$$

where $(.)^{\dagger}$ stands for the Moore-Penrose pseudo-inverse. The transformation matrix $\mathbf{B}$, however, is usually not capable of transforming the response perfectly across the entire sector $\mathcal{S}$ except for the case where a large number of antenna elements is present or a very small sector is used. The error of the transform is defined as

$$
\epsilon(\mathcal{S})=\frac{\left\|\overline{\mathbf{A}}_{\mathcal{S}}-\mathbf{B} \mathbf{A}_{\mathcal{S}}\right\|_{\mathrm{F}}}{\left\|\overline{\mathbf{A}}_{\mathcal{S}}\right\|_{\mathrm{F}}} \in \mathbb{R}^{+} .
$$

Large transformation errors often result in a large bias on the final DOA estimations, thus, usually, the response region is divided into a set of regions called sectors, and a different transform matrix is set up for each sector (sector-by-sector processing).

\section{PROPOSED APPROACH}

In this section a signal adaptive approach for array interpolation is shown and detailed in Subsections 3.1, 3.2 and 3.3.

\subsection{Power Scanning and Sector Selection}

Since the array response needs to be known to construct $\mathbf{B}$, such knowledge can be used to detect angular regions where significant power is received. This estimation can be done with the conventional beamformer [18], yielding the normalized power response

$$
P(\theta)=\frac{\mathbf{a}^{\mathrm{H}}(\theta) \hat{\mathbf{R}}_{\mathbf{X X}} \mathbf{a}(\theta)}{\mathbf{a}^{\mathrm{H}}(\theta) \mathbf{a}(\theta)} \in \mathbb{R},
$$

where $\hat{\mathbf{R}}_{\mathbf{X X}}=\frac{\mathbf{X X}^{\mathrm{H}}}{N}$ is the estimate of the signal covariance matrix. In real systems the result of (7) is discrete in $\theta$ and can be written as

$$
P[z]=P\left(-90^{\circ}+(z \cdot \Delta)\right)=P(\theta),
$$

with $\theta \in \mathcal{D}_{\Delta}$ where

$$
\mathcal{D}_{\Delta}=\left\{-90^{\circ},-90^{\circ}+\Delta, \ldots, 90^{\circ}-\Delta, 90^{\circ}\right\}
$$

and $\Delta$ is the resolution of the azimuth angle of the power response (7).

The output of (7) is scanned for sectors, and for each sector the respective lower bound $l_{k} \in \mathcal{D}_{\Delta}$ and upper bound $u_{k} \in \mathcal{D}_{\Delta}$ are defined as shown in Figure 1. The threshold that defines a sector and its

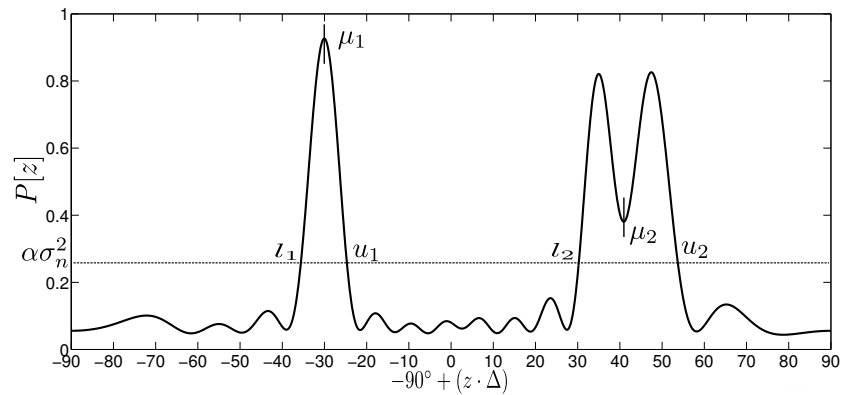

Fig. 1: Selected sectors and respective bounds

bounds can be defined, for instance, by looking at the noise power. The noise floor can be set at $\alpha \sigma_{n}^{2}$, with $\alpha>1$ being a sensitivity parameter. A large $\alpha$ means that only large sectors (large detected amplitude) are detected but coming at the cost of discarding smaller (smaller detected amplitude) sectors that are related to a signal component, while a small $\alpha$ means that smaller sectors are detected but at the cost of allowing noise to be mistakenly detected as a sector. If $K$ sectors are detected, a detected sector with bounds $\left[l_{k}, u_{k}\right]$ is said to be centered at

$$
\mu_{k}=\left\lceil\frac{\left|u_{k}-l_{k}\right|}{2}\right\rfloor_{\mathcal{D}_{\Delta}} \in \mathcal{D}_{\Delta},
$$


where $\lceil\cdot\rfloor_{\mathcal{D}_{\Delta}}$ is a rounding operation to the domain $\mathcal{D}_{\Delta}$. A weighting factor for each sector is calculated as

$$
\xi_{k}=\frac{\sum_{z=l_{k}}^{u_{k}} P[z]}{\sum_{w=1}^{K} \sum_{z=l_{w}}^{u_{w}} P[z]} \in \mathbb{R} .
$$

As mentioned in Section 2, classical array interpolation methods in the literature divide the array response into various sectors (sectorby-sector processing) in order to keep the error (6) small. However, in this work a single transform matrix is used based on a combination of the sectors detected in (7). In order to bound the error $\epsilon(\mathcal{S})$ a signal adaptive method for calculating the maximum transform size is used based on the weights calculated in (11). For a sector centered at $\mu_{k}$, the discrete and countable set of angles used to transform this sector is given by

$$
\mathcal{S}_{k}= \begin{cases}\left\{\left\lceil\mu_{k}-\frac{\Xi \xi_{k}}{2}\right]_{\mathcal{D}_{\Delta}},\left[\mu_{k}-\frac{\Xi \xi_{k}}{2}+\Delta\right]_{\mathcal{D}_{\Delta}}, \ldots,\left[\mu_{k}+\frac{\Xi \xi_{k}}{2}\right]_{\mathcal{D}_{\Delta}}\right. & , \Xi \xi_{k}<\left|u_{k}-l_{k}\right| \\ \left\{l_{k}, l_{k}+\Delta, \ldots, u_{k}, \Xi \xi_{k}\right\} \geq\left|u_{k}-l_{k}\right| & \end{cases}
$$

and

$$
\mathcal{S}_{k} \cap \mathcal{S}_{\bar{k}}=\emptyset \forall k, \bar{k} \in\{1, \ldots, K\} \text { and } \mathrm{k} \neq \overline{\mathrm{k}},
$$

where $\Xi \in \mathbb{R}^{+}$is the total transform size in degrees of all sectors. The combined sector is given by

$$
\mathcal{S}=\mathcal{S}_{1} \cup \mathcal{S}_{2} \cup \ldots \cup \mathcal{S}_{K}
$$

Thus, $\mathcal{S}$ has a "wider" support for the sectors $\mathcal{S}_{k}$ where more power is present (weighted by $\xi_{k}$ ), i.e the transformation of the combined sector $\mathcal{S}$ is weighted towards the sectors $\mathcal{S}_{k}$ that include more signal power.

As the problem of obtaining the transform matrix $\mathbf{B}$ is equivalent to solving a highly overdetermined system we have

$$
|\mathcal{S}| \rightarrow \infty \Longleftrightarrow \epsilon(\mathcal{S}) \rightarrow \infty \text {. }
$$

Thus, transforming the entire detected sectors may result in a very high transformation error introducing a very large bias into the final DOA estimates. To address this problem an upper bound to the transform error $\epsilon_{\max }$ needs to be defined and a search can be performed to find the maximum transform size covering the detected sectors that is still within the error upper bound. The problem of finding the maximum $\Xi$ with respect to $\epsilon_{\max }$ can be written as the optimization problem

$$
\begin{gathered}
\max _{\Xi} \epsilon(\mathcal{S}) \\
\text { subject to } \epsilon(\mathcal{S}) \leq \epsilon_{\max } \\
\Xi \leq \Xi_{\max }=\sum_{k=1}^{K}\left|u_{k}-l_{k}\right| \\
\Xi \geq \Xi_{\min }=M \Delta .
\end{gathered}
$$

The problem in (16)-(19) can efficiently be solved using a bisection search method since, once all $\mu_{k}$ have been defined, the error function increases monotonically for $\Xi>\Xi_{\text {min }}$, as illustrated in Figure 2. $\epsilon(\mathcal{S})$ is greatly affected if the calculation of $\mathbf{B}$ is either a heavily overdetermined or an underdetermined system. Therefore, $\Xi_{\min }$ is defined to ensure monotonicity of the problem given in (16).

\subsection{Data Transformation}

Once (16) has been solved and $\mathbf{B}$ has been calculated according to (5) the transformed covariance including FBA and SPS is obtained

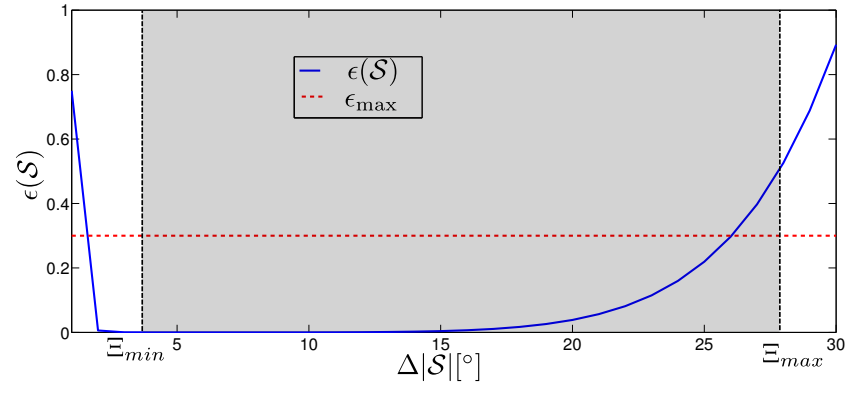

Fig. 2: Transformation error with respect to combined sector size

by [7]

$$
\overline{\mathbf{R}}_{\mathbf{X X}}=\frac{1}{2 L} \sum_{l=1}^{L} \mathbf{J}_{l}^{\mathrm{T}}\left(\mathbf{B} \hat{\mathbf{R}}_{\mathbf{X X}} \mathbf{B}^{\mathrm{H}}+\mathbf{Q B} \hat{\mathbf{R}}_{\mathbf{X} \mathbf{x}}^{*} \mathbf{B}^{\mathrm{H}} \mathbf{Q}\right) \mathbf{J}_{l},
$$

where $(\cdot)^{*}$ stands for the complex conjugation, $\mathbf{Q}$ is a matrix containing ones in its anti-diagonal and zeros elsewhere, $\mathbf{J}_{l}$ is an appropriate selection matrix responsible for selecting the sub-arrays to be averaged, and $L$ is the total number of sub-arrays employed. While $L$ can be chosen a priori it can also be adaptively chosen as to minimized the loss of effective array aperture while achieving a good estimate of $d$. We use as a model order estimation (MOE) method $\operatorname{MOE}\left(\overline{\mathbf{R}}_{\mathbf{X X}}(L)\right)=\hat{d}$ the RADOI [19]. Therefore, we have to solve the problem

$$
(L, \hat{d})=\arg \min _{L} \max _{\hat{d}}\left\{\operatorname{MOE}\left(\overline{\mathbf{R}}_{\mathbf{X X}}(L)\right)\right\} .
$$

For multidimensional problems more accurate methods [20], [21] can be used. It is important to notice that the estimated number of impinging signals $\hat{d}$ can be different from the number of sectors detected in (7). Each of the detected sectors $\mathcal{S}_{k}$ can be formed by a set of nearly coherent signals that now can be efficiently separated with (21) allowing the application of a high resolution DOA estimation method to jointly estimate the parameters of all the detected signals.

\subsection{GEVD and ESPRIT}

After solving the problem in (21) a joint high resolution estimate of the DOAs can be obtained, as shown in [22], by applying the GEVD on the FBA-SPS covariance matrix $\overline{\mathbf{R}}_{\mathbf{X X}}$.

$$
\overline{\mathbf{R}}_{\mathrm{XX}} \mathbf{E}=\overline{\mathbf{R}}_{\mathrm{NN}} \mathbf{E} \boldsymbol{\Lambda},
$$

where $\mathbf{E} \in \mathbb{C}^{M \times M}$ is a matrix containing the generalized eigenvectors and $\boldsymbol{\Lambda} \in \mathbb{R}^{M \times M}$ is a matrix containing the generalized eigenvalues in its diagonal. By selecting the eigenvectors related to the $\hat{d}$ largest eigenvalues the so called signal subspace $\mathbf{E}_{s} \in \mathbb{C}^{M \times \hat{d}}$ is constructed. This signal subspace needs to be dewhitened or projected back onto the original response subspace prior to estimation, this can be done by

$$
\overline{\mathbf{E}}_{s}=\overline{\mathbf{R}}_{\mathrm{NN}} \mathbf{E}_{s} \text {. }
$$

With this subspace estimate at hand the Total Least Squares (TLS) ESPRIT [14] is applied. For multidimensional arrays another option is to employ methods based on the PARAFAC decomposition such as [23], [24].

Finally, from the first set of estimates the VIT [17] can be applied followed by $\hat{d}$ extra applications of ESPRIT to obtain more precise estimates. 


\section{NUMERICAL SIMULATIONS AND DISCUSSION}

To test the efficiency of the proposed approach as defined in Subsections 3.1, 3.2, and 3.3 a set of numerical simulations is performed and results are compared to the Cramér-Rao bound (CRB) for the true array response $\mathbf{a}(\theta)$ and compared to the approach proposed in [12]. In Subsection 4.1 the performance of the proposed method is studied in the presence of spatially white Gaussian noise. In Subsection 4.2 the case where the measurement data of the array response contains errors is studied.

\subsection{Performance in the presence of white noise}

The array response assumed in the simulations shown in Figure 3 is constructed by randomly displacing the elements of a Uniform Linear Array (ULA) composed of $M=8$ antennas with inner element spacing of $\frac{\lambda}{2}$ to a point belonging to a circle with center on the original antenna position and radius $\frac{0.1 \lambda}{2}$, where $\lambda$ is the wavelength of the carrier frequency of the signal. For obtaining $\hat{\mathbf{R}}_{\mathbf{X X}}$ we use $N=200$ snapshots and the Root Mean Squared Error (RMSE) is calculated with respect to 1000 Monte Carlo simulations. Three signals impinging from $\theta_{1}=45^{\circ}$, $\theta_{2}=38^{\circ}$ and $\theta_{3}=15^{\circ}$ with $\sigma_{1}^{2}=\sigma_{2}^{2}=\sigma_{3}^{2}=1$ and $\gamma_{1,2}=1, \gamma_{2,3}=\gamma_{1,3}=0.8$ according to equation (4) are impinging on the array. The Signal to Noise Ratio (SNR) is defined as $\mathrm{SNR}=\frac{\sigma_{1}^{2}}{\sigma_{\mathrm{n}}^{2}}=\frac{\sigma_{2}^{2}}{\sigma_{\mathrm{n}}^{2}}=\frac{\sigma_{3}^{2}}{\sigma_{\mathrm{n}}^{2}}$. In Figures 3 and 4 the given RMSE is $\operatorname{RMSE}=\sqrt{\frac{1}{K} \sum_{k=1}^{K}\left(\left(\hat{\theta}_{1, k}-\theta_{1}\right)^{2}+\left(\hat{\theta}_{2, k}-\theta_{2}\right)^{2}+\left(\hat{\theta}_{3, k}-\theta_{3}\right)^{2}\right)}$, where $\hat{\theta}_{i}$ is the estimate of $\theta_{i}$. The CRB shown in Figure 3 is the sum CRB for all estimated DOAs. The two parameters that fully define the proposed approach given in Subsections 3.1, 3.2, and 3.3 are set to $\alpha=1.2$ and $\epsilon_{\max }=10^{-3}$.

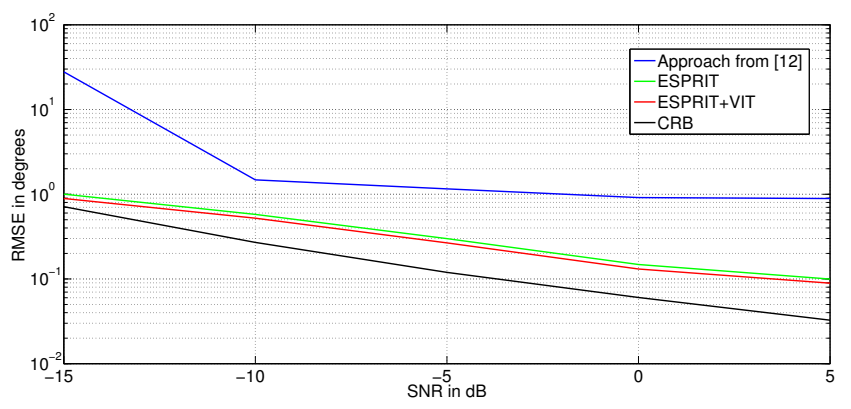

Fig. 3: RMSE for [12], ESPRIT and ESPRIT+VIT

In [12] a sector-by-sector processing with out-of-sector response filtering and applying MUSIC is proposed. We use the same approach for the calculation of the transformation matrices as given in [12] while additionally applying FBA and SPS as well as using Root-MUSIC instead of MUSIC. Furthermore, we assumed, for the simulations of the approach given in [12] that the model order is perfectly known. Figure 3 shows that the estimates provided by the approach of [12] with Root-MUSIC quickly reach a plateau for this demanding signal scenario as the DOA estimation bias is dominated by large transformation errors for each sector. On the other hand, the approach proposed in this work provides improved accuracy with increasing SNR since the size of the combined sector also decreases, resulting in a much smaller transformation error. The proposed approach is still not capable of reaching the CRB due to the application of SPS, which effectively decreases array aperture, however, $L$ being chosen according to (21).

\subsection{Robustness to Errors in the Array Response Model}

In reality the true array response is not fully known and can only be derived by empirical measurements which include measurement errors. To study a scenario where the measurements data of the array response are corrupted by measurement errors we introduce additional positioning errors. We consider the same signal scenario as in Section 4.1 while the SNR for this set of simulations is kept fixed at $15 \mathrm{~dB}$ and the variance $\sigma_{e}^{2}$ of the additional errors are given in fractions of $\frac{\lambda}{2}$ and range from 0.02 to 0.2 .

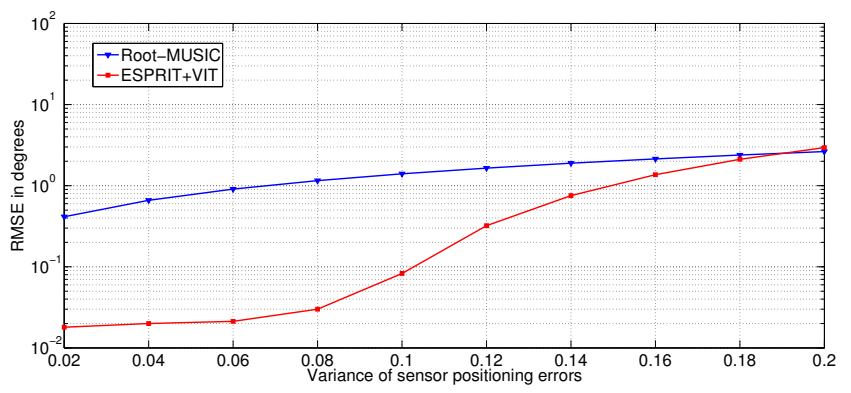

Fig. 4: RMSE with array response model errors

Figure 4 shows that the proposed approach provides improved accuracy for errors drawn from $\mathcal{N}\left(0, \sigma_{e}^{2}<0.2\right)$. Since the proposed approach relies on the shift invariance of the array, arrays with large unknown positioning errors (errors of empirical measurements) are not correctly transformed into shift invariant arrays, thus, resulting in large inaccuracies in the final estimations.

\section{CONCLUSION}

In this work a novel, low complexity, signal adaptive method for DOA estimation with interpolated arrays is presented. The received signal is used to obtain a single transform matrix instead of the traditional sector-by-sector processing. The closed form ESPRIT together with the VIT is applied without the need of explicitly prewithening the transformed covariance and the joint estimation of all waveforms avoids the problem of selecting different sector estimates (double estimates or "ghost" signals). The proposed approach can easily be extended for multidimensional signal processing for arrays with arbitrary array geometry. The proposed approach achieves improved accuracy in DOA estimation with respect to state of the art methods and provides robustness to errors in the array response model. ${ }^{1}$

\footnotetext{
${ }^{1}$ The research leading to the results reported in this paper has received funding from the European Community's Seventh Framework Programme (FP7/2007-2013) under grant agreement $n^{\circ} 287207$ as well as funding from the German Federal Ministry of Economics and Technology under grant agreement 50NA1110 and Fundação de Apoio a Pesquida do Distrito Federal (FAPDF). This support is greatly acknowledged.
} 


\section{REFERENCES}

[1] Y. Bresler and A. Macovski, "Exact Maximum Likelihood Estimation of Superimposed Exponentials Signals in Noise," IEEE ASSP Magazine, vol. 34, pp. 1081-189, 1986.

[2] P. Stoica and A. Nehorai, "A Novel Eigenanalysis Method for Direction Estimation," in Proceedings IEEE F., 1990.

[3] A. J. Barabell, "Improving the Resolution Performance of Eigenstructured Based Direction-Finding Algorithms," in Proceedings of ICASSP 83, 1983.

[4] J. E. Evans, J. R. Johnson, and D. F. Sun, "Application of advanced signal processing techniques to angle of arrival estimation in ATC navigation and surveillance system," Massachusetts Institute of Technology, Tech. Rep., 1982.

[5] S. Pillai and B. H. Kwon, "Forward/backward Spatial Smoothing Techniques for Coherent Signal Identification ," IEEE Transactions on Acoustics, Speech and Signal Processing, vol. 37, pp. 8-9, January 1989.

[6] T. Bronez, "Sector interpolation of non-uniform arrays for efficient high resolution bearing estimation," in Acoustics, Speech, and Signal Processing, 1988. ICASSP-88., 1988 International Conference on, 1988.

[7] B. Friedlander and A. Weiss, "Direction finding using spatial smoothing with interpolated arrays," Aerospace and Electronic Systems, IEEE Transactions on, vol. 28, pp. 574-587, 1992.

[8] B. Friedlander, "The root-MUSIC algorithm for direction finding with interpolated arrays," Signal Processing, vol. 30, pp. 15-29, 1993.

[9] M. Pesavento, A. Gershman, and Z.-Q. Luo, "Robust array interpolation using second-order cone programming," Signal Processing Letters, vol. 9, pp. 8-11, 2002.

[10] M. Buhren, M. Pesavento, and J. F. Böhme, "Virtual array design for array interpolation using differential geometry," in Acoustics, Speech, and Signal Processing, 2004. Proceedings. (ICASSP '04). IEEE International Conference on, vol. 2, 2004.

[11] B. Lau, G. Cook, and Y. Leung, “An improved array interpolation approach to DOA estimation in correlated signal environments," in Acoustics, Speech, and Signal Processing, 2004. Proceedings. (ICASSP '04). IEEE International Conference on, 2004.

[12] B. Lau, M. Viberg, and Y. Leung, "Data-adaptive array interpolation for DOA estimation in correlated signal environments," in Acoustics, Speech, and Signal Processing, 2005. Proceedings. (ICASSP '05). IEEE International Conference on, 2005.

[13] R. O. Schmidt, "Multiple emitter location and signal parameter estimation," IEEE Transactions on Antennas and Propagation, vol. 34, pp. 276-280, 1986.

[14] R. Roy and T. Kailath, "ESPRIT - estimation of signal parameters via rotation invariance techniques," IEEE Transactions on Acoustics Speech and Signal Processing, vol. 17, 1989.

[15] M. Buhren, M. Pesavento, and J. F. Bohme, “A new approach to array interpolation by generation of artificial shift invariances: interpolated ESPRIT," in Acoustics, Speech, and Signal Processing, 2003. Proceedings. (ICASSP '03). 2003 IEEE International Conference on, 2003.
[16] A. Weiss and M. Gavish, "The interpolated ESPRIT algorithm for direction finding," in Electrical and Electronics Engineers in Israel, 1991. Proceedings., 17th Convention of, 1991.

[17] T. Kurpjuhn, M. Ivrlac, and J. Nossek, "Vandermonde Invariance Transformation," in Acoustics, Speech, and Signal Processing, 2001. Proceedings. (ICASSP '01). 2001 IEEE International Conference on, 2001.

[18] M. Bartlett, "Smoothing Periodograms from Time Series with Continuous Spectra," Nature, vol. 161, pp. 686-687, 1948.

[19] E. Radoi and A. Quinquis, "A new method for estimating the number of harmonic components in noise with application in high resolution radar," EURASIP Journal on Applied Signal Processing, pp. 1177-1188, 2004.

[20] J. P. C. L. da Costa, M. Haardt, F. Romer, and G. Del Galdo, "Enhanced model order estimation using higher-order arrays," Conference Record of The Forty-First Asilomar Conference on Signals, Systems \& Computers, pp. 412-416, 2007.

[21] J. P. C. L. da Costa, F. Roemer, M. Haardt, and R. T. de Sousa Jr., "Multi-Dimensional Model Order Selection," EURASIP Journal on Advances in Signal Processing, vol. 26, 2011.

[22] M. Haardt, R. S. Thoma, and A. Richter, "Multidimensional high-resolution parameter estimation with applications to channel sounding," High-Resolution and Robust Signal Processing, pp. 255-338, 2004.

[23] J. P. C. L. da Costa, D. Schulz, F. Roemer, M. Haardt, and J. A. A. Jr., "Robust R-D Parameter Estimation via ClosedForm PARAFAC in Kronecker Colored Environments," in Proc. 7-th International Symposium on Wireless Communications Systems (ISWCS 2010), 2010.

[24] J. P. C. L. da Costa, F. Roemer, M. Weis, and M. Haard, "Robust R-D parameter estimation via closed-form PARAFAC," in Proc. ITG Workshop on Smart Antennas (WSA'10), 2010. 